\title{
Random sequential adsorption with two components: asymptotic analysis and finite size effects
}

\author{
Louise Reeve and Jonathan A.D. Wattis \\ School of Mathematical Sciences, University of Nottingham, University Park, \\ Nottingham NG7 2RD, UK \\ E-mail: pmylr3@nottingham.ac.uk, Jonathan.Wattis@nottingham.ac.uk
}

\begin{abstract}
We consider the model of random sequential adsorption (RSA) in which two lengths of rod-like polymer compete for binding on a long straight rigid onedimensional substrate. We take all lengths to be discrete, assume that binding is irreversible, and short or long polymers are chosen at random with some probability. We consider both the cases where the polymers have similar lengths and when the lengths are vastly different. We use a combination of numerical simulations, computation and asymptotic analysis to study the adsorption process, specifically, analysing how competition between the two polymer lengths affects the final coverage, and how the coverage depends on the relative sizes of the two species and their relative binding rates. We find that the final coverage is always higher than in the one-species RSA, and that the highest coverage is achieved when the rate of binding of the longer polymer is higher. We find that for many binding rates and relative lengths of binding species, the coverage due to the shorter species decreases with increasing substrate length, although there is a small region of parameter space in which all coverages increase with substrate length.
\end{abstract}

Keywords: Random sequential adsorption, asymptotic analysis, recurrence relations. PACS: $68.43 . \mathrm{Mn}$ Kinetics of adsorption, 64.60.-i general studies of phase transitions, 64.60.an finite size systems

Submitted to: J. Phys. A: Math. Gen.

\section{Introduction}

Random Sequential Adsorption (RSA) is a model that has been of interest since the 1939 paper by Flory [11] and 1958 paper by Rényi [24]. Due to its many applications, RSA is of wide interest in many fields of science, including physics $[5,7,16]$, biology [8], pharmacy [20, 23] chemistry [1, 17] and computer science [21]. Evans [9] gives an extensive review of the topic. Talbot et al. [27] also review sequential adsorption processes. As well as covering $1 \mathrm{D}$ and $2 \mathrm{D}$ cases, they discuss many of the additional 
effects which complicate such processes, with particular reference to applications in colloidal systems.

An important technique in gene therapy is to deliver DNA into an defective cell's nucleus, which can then go on to create a functional protein to treat a disease. DNA has many features which cause it to be spatially extended, one being the electrostatic repulsion between phosphates on its backbone. For DNA to be condensed, these negative charges must be neutralised. The original application motivating this present work is that of different lengths of positively charged polymers attaching to a negatively charged DNA to neutralise its charge, allowing it to condense and be transported into the cell's nucleus. In experimental work, it has been found that DNA condenses when it reaches $90 \%$ neutralisation [12]. Since it is possible to design polymers with specific properties, our motivation is to design polymer mixtures to maximise coverage.

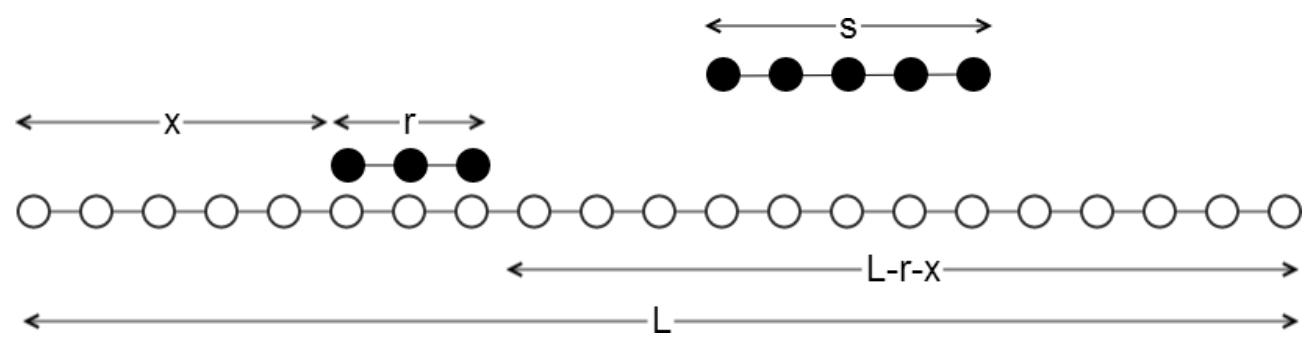

Figure 1. Diagram of polymer of length $r$ bound to the substrate of length $L$, and a polymer of length $s$ attempting to bind in the remaining gap of length $L-r-x$

RSA is also known as the "Parking Problem" due to the similarity with trying to park a car on a street. This can be modelled in two ways: discrete or continuous substrates, here we focus on the discrete case. Our aim in this paper is to analyse random sequential adsorption (RSA) processes in which two distinct lengths of 'polymer' $(r, s)$ which are assumed to be straight and rigid bind to a long straight rigid one-dimensional substrate, of length $L$ (with $L \gg r, s$ ) as summarised in figure 1. We are concerned with how the coverage $(\theta$, with $0 \leq \theta \leq 1)$ depends on the length of the polymers concerned, and in particular, finite size effects.

In discrete RSA, the substrate has a discrete number of unit binding sites to which a polymer can bind. Where one polymer lands affects where others can land, as we assume that they cannot be removed once bound, and cannot overlap. Our main interest is the total coverage achieved by the polymers, that is, the proportion of the substrate occupied by polymers. Cornette et al. [6] show that total coverage approaches jamming exponentially in time. However, the jamming limit is not straightforward, as Bonnier et al [3] analyse the pair correlation function for the adsorbed sites, revealing complex structure in the jammed state.

Clearly this basic formulation of RSA has made a number of simplifications which do not hold in many practical applications. For example, a more accurate model of polymer binding to DNA would allow both the DNA substrate and the adsorbing polymers to be flexible, and allow the adsorption to occur in three-dimensional space. A large range 
of generalised RSA systems have been covered: Tarjus et al. [26] have considered the effect of adding desorption and diffusion of adsorbed species on the substrate to RSA. They consider hard spheres being adsorbed onto a flat 2D surface. They use distribution functions to describe the kinetics of the process which, instead of the jamming limit, now approaches a dynamic equilibrium in which desorption rates balance adsorption. Maltsev et al. [19] consider reversible adsorption and motion on the substrate, in the case of a discrete one-dimensional substrate. In this simpler system, it is possible to analyse how the distribution of gaps between adsorbed polymers changes over time, and so describe the kinetics of adsorption. The addition of small rate of desorption allows higher coverages to be attained than in pure RSA.

Complexity above one-dimensional, but below two-dimensional have been considered by Baram and Kutasov [2], who consider a $2 \times \infty$ lattice. By using a master equation approach, they show that the jamming density is $\frac{1}{2}\left(1-\frac{1}{2} \mathrm{e}^{-1}\right)=0.408$, significantly less than the one-dimensional case, which for dimers landing on a $1 \mathrm{D}$ discrete substrate gives $\phi_{d}=1-\mathrm{e}^{-2}=0.86466$, or for unit particles adsorbing onto a continuous substrate gives Rényi's constant, $\phi_{c}=0.7476$. Feder's approximation for dimers on a two-dimensional substrate gives $\phi_{c}^{2}=0.5589$ or $\phi_{d}^{2}=74765$ in the cases of continuous and discrete substrate respectively.

Various aspects of the case where the binding species includes a mixture of lengths have been considered by Hassan and Kurths [13], Hassan et al [14], Burridge and Mao [4], and Purves et al. [22]. Hassan and Kurths [13], study the two-component RSA system with a continuous substrate. This is simplified by making one of the two binding species have zero length. They seek solutions for the PDEs describing the gap distribution in terms of a self-similar solution, obtaining the distribution of the form $t^{2} \mathrm{e}^{-x t}$. A similar system is studied by Hassan et al [14], where now both species have finite length, $\sigma$ and $m \sigma$ with $1<m \leq 2$. After deriving the PDEs for the evolution of the gap length distribution, they present numerical results showing the total coverage against the probability of the shorter polymer binding. This is paper is the closest in spirit to the work we present below in Sections 2 and 3. Burridge and Mao [4] consider RSA in which the lengths of adsorbing species are drawn randomly from a distribution, again with the restriction that the longest possible polymer has to be less than twice the length of the shortest. They find exact analytic formulae for the coverage, whilst these expressions are confirmed against numerical simulations, they are too complicated to evaluate analytically.

Purves et al. [22] consider the scaling of coverage with availability of binding sites $(A)$ in the jamming limit. They consider the case of two distinct lengths of binding species, hence there are two availabilities, $A_{r}(t)$ for the shorter species and $A_{s}(t)$ for the longer. In the case of a discrete substrate, $A_{r}(t) \propto \theta(\infty)-\theta(t)$, and $A_{s}(t) \propto$ $[\theta(\infty)-\theta(t)]^{n}$ where $n$ depends on the relative rate of binding of the two species and the difference in their lengths. For the continuous substrate, $A_{r}(t) \propto[\theta(\infty)-\theta(t)]^{2}$ and $A_{s}(t) \propto[\theta(\infty)-\theta(t)]^{2} \exp -\kappa /[\theta(\infty)-\theta(t)]$. These scaling laws carry over to the twodimensional system, where simulations of square blocks landing on a two-dimensional 
substrate give $A \propto \theta(\infty)-\theta(t)$ for the discrete substrate and $A \propto[\theta(\infty)-\theta(t)]^{2}$ for the continuous.

Feder [10] simulated the sequential filling of a surface by disks, and obtained the limit of 0.55 which is close to the square of Rényi's constant. Hinrichsen et al. [15] perform simulations of disks and line segments onto a two-dimensional substrate, obtaining the hole size distribution and coverage in the jamming limit. Viot et al. [28] consider the jamming limit for the case of various shapes of $2 \mathrm{D}$ objects being adsorbed onto a $2 \mathrm{D}$ surface. The coverage approaches its limit in algebraic fashion, namely $\theta(\infty)-\theta(t) \sim t^{-n}$, with an exponent $(n)$ that depends on the shape of the object being adsorbed. Whilst circular, and most other shaped objects show a standard asymptotic behaviour with $n=1 / 3$, they find that for highly elongated shapes, they find the exponent $n=1 / 2$, together with cross-over behaviour between this exponent and the standard asymptotic behaviour. Talbot and Schaaf [25] consider the adsorption onto a continuous two-dimensional substrate of a two-component mixture of circular disks with vastly differing sizes. By considering exclusion circles around each adsorbed disc, they deriving ODEs for the coverage due to each size of disc. These yield the approximate behaviour of the system in the jamming limit, and in the case of the small discs becoming vanishingly small, the results are highly accurate.

\begin{tabular}{c|c|l} 
Variable & Typical value & Description \\
\hline$L$ & $10^{4}$ & Length of substrate $(L \gg 1)$ \\
$r$ & $2 \leq r \leq 10$ & Length of shorter polymer \\
$s$ & $5 \leq s \leq 100$ & Length of longer polymer $(s>r)$ \\
\hline$p$ & $0 \leq p \leq 1$ & Probability of the short polymer being chosen for binding \\
$t$ & $t<10^{4}$ & Number of binding attempts (a proxy for time) \\
\hline$N(L)$ & $N<L / r$ & Number of polymers attached to the substrate \\
$N_{r}(L)$ & $N_{r}<L / r$ & Number of short polymers attached to the substrate \\
$N_{s}(L)$ & $N_{s}<L / s$ & Number of long polymers attached to the substrate \\
\hline$\theta$ & $0<\theta<1$ & Total coverage \\
$\theta_{r}$ & $0<\theta_{r}<1$ & Coverage by the shorter polymers \\
$\theta_{s}$ & $0<\theta_{s}<1$ & Coverage by the longer polymers \\
\hline
\end{tabular}

Table 1. Summary of parameters, variables and notation used in this paper.

In the remainder of this section, we recap the results from the one-component case of RSA. The notation used throughout this paper is summarised in Table 1. Section 2 contains our theoretical results on the expected outcome of the two component case. In section 3 we present the results obtained from Monte Carlo (MC) simulations of the system, together with a numerical solution of a deterministic formulation of the problem (Section 2), which gives the expected outcome of the MC simulations. In section 4 we derive the solutions using asymptotic methods for the substrate being much longer than the polymers; we consider two distinct cases; (i) when the polymers have similar lengths, and (ii) when one polymer is significantly longer than the other, whilst both are much 
shorter than the substrate. Conclusions are drawn and discussed in section 5 .

\subsection{One polymer case}

The one-polymer formulation of RSA was first proposed by Rényi [24]; in this model, the substrate is continuous and the expected density of cars parked on a street of infinite length is $74.75979 \%$. For the case of a discrete substrate, the coverage depends on the length of binding species. Here we summarise the averaged results for the case of a single species of length $r$ binding to a discrete substrate of length $L$.

To form the recurrence relation, we first observe that when the substrate length, $L$, is less than that of the polymer, $r$, no polymers can bind. When $L$ is between $r$ and $2 r$, only one polymer is able to bind. Once the length of the substrate reaches and exceeds $2 r$, the number of polymers bound, $N_{r}$, depends on the location that each prior polymer has landed on the substrate. This is because the gap each side of the polymer could have size greater than or less than $r$. The equations governing jamming coverage are thus

$$
\begin{aligned}
& N(L)=0, \quad(L<r), \\
& N(L)=1, \quad(r \leq L<2 r), \\
& N(L)=1+\frac{2}{(L-r+1)} \sum_{n=r}^{L-r} N(n), \quad(L \geq 2 r) .
\end{aligned}
$$

Rearranging the latter equation yields

$$
(L-r+1)[N(L)-1]=2 \sum_{n=r}^{L-r} N(n) .
$$

Now we apply the transformation $L \rightarrow L+1$ and take the difference of the resulting equation and (4) to obtain the recurrence relation

$$
(L-r+2) N(L+1)=(L-r+1) N(L)+2 N(L-r+1)+1 .
$$

The coverage, $\theta$, is defined to be the proportion of substrate sites that have polymer bound, and is calculated by $\theta(t)=r N(L) / L$.

The first graph in figure 2 shows that once the substrate length passes a certain length, the relationship formed between the number of polymers bound and the substrate length is approximately linear. The second graph shows that, again after the substrate reaches a certain length, the coverages asymptote to just below $80 \%$ coverage. This value corresponds to the gradient of the curve in the first panel.

\section{Two polymer case}

In the case where polymers of two distinct lengths can bind to the substrate, we require two variables, $N_{r}$ and $N_{s}$ to describe the RSA process. We are interested in how the coverage is affected by each polymer length, and by the relative rate of binding of the two 

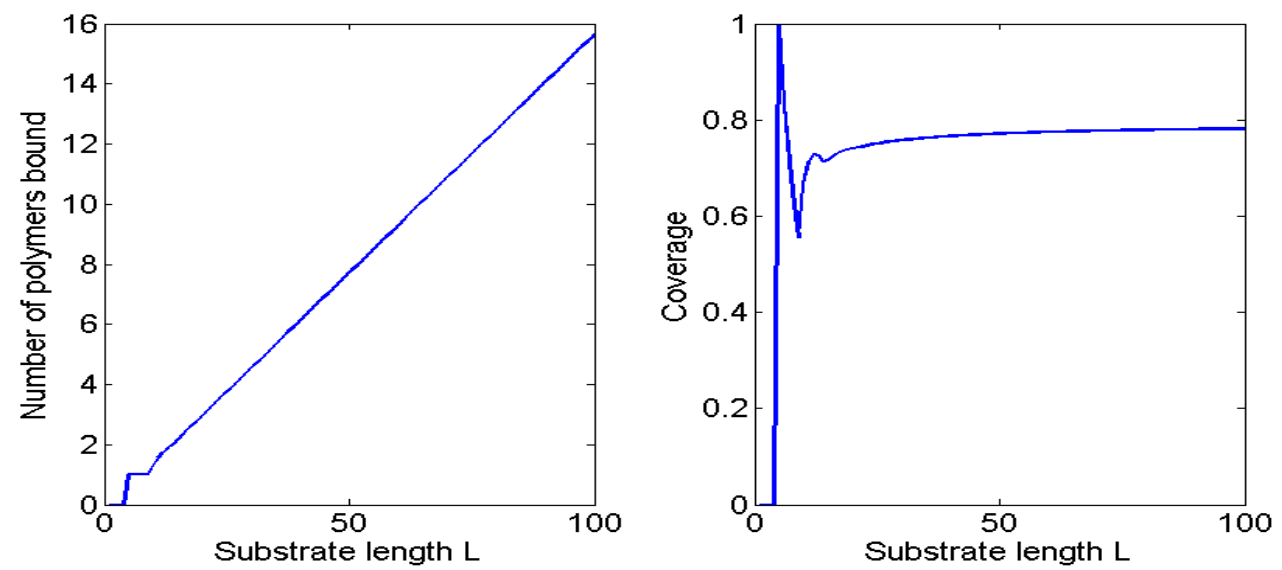

Figure 2. Graphs obtained using the recurrence relation (5) for the one polymer case when $r=5$. Left, the number of polymers bound to the substrate as the length of the substrate varies. Right, the coverage by the polymers as substrate length varies.

polymers. We denote the two lengths of polymer by $r$ and $s$, and use $p$ for the probability of the shorter polymer $(r)$ binding, so that the probability of the longer polymer $(s>r)$ binding is $1-p$. Clearly, a stochastic process will give different coverages on each realisation; however, averaging over a large number of simulations leads to well-defined values for the coverage due to each species. We consider the kinetics of the binding process, using a time unit corresponding to the number of binding attempts. Using an alternative notation, we could define binding rates $K_{r}$ for the $r$-polymers and $K_{s}$ for the $s$-polymers, then $p=K_{r} /\left(K_{r}+K_{s}\right)$.

To understand the expected coverage of the stochastic process, we assume there is no cooperativity between the binding of short and short, or short and long, or long and long polymers, that is, each binding event is independent of the arrangement of polymers already bound to the substrate. We also assume that there is no specific spatial arrangement of bound polymers, so that it is sufficient to keep track of the total numbers of each type of polymer on the substrate, that is, a spatially averaged description of the substrate will suffice. These assumptions are similar to those made in the construction of 'mean field' models; hence the system of equations that we derive can be considered as mean field model of RSA. Whilst both $N_{r}, N_{s}$ are functions of $L$, $r, s$ and $p$, we consider $r, s$, and $p$ as fixed parameters, and focus on describing how $N_{r}, N_{s}$ depend on the substrate length variable, $L$. Firstly, we formulate equations for each of $N_{r}(L), N_{s}(L)$ when just the $r$-polymer binds

$$
N_{r}(L)=1+\frac{2}{(L-r+1)} \sum_{n=0}^{L-r} N_{r}(n), \quad N_{s}(L)=0+\frac{2}{(L-r+1)} \sum_{n=0}^{L-r} N_{s}(n), \quad(L \geq r) .
$$

Secondly, if we assume instead that a polymer of length $s$ binds, we have the recurrence 
relations

$$
N_{r}(L)=0+\frac{2}{(L-s+1)} \sum_{n=0}^{L-s} N_{r}(n), \quad N_{s}(L)=1+\frac{2}{(L-s+1))} \sum_{n=0}^{L-s} N_{s}(n), \quad(L \geq s) .
$$

As well as the clear change in placement of the zero and one terms on the RHSs, equations (6) and (7) differ in the denominator and the limits on the sums.

Multiplying (6) and (7) by $p(L-r+1)$ and $(1-p)(L-s+1)$, respectively, and adding, we obtain equations for the full process in which polymers are chosen at random to bind, namely

$$
\begin{aligned}
\Lambda N_{r}(L)= & p(L-r+1)+2 p \sum_{n=0}^{L-r} N_{r}(n)+2(1-p) \sum_{n=0}^{L-s} N_{r}(n), \\
\Lambda N_{s}(L)= & (1-p)(L-s+1)+2 p \sum_{n=0}^{L-r} N_{s}(n)+2(1-p) \sum_{n=0}^{L-s} N_{s}(n), \\
& \text { for } L \geq s, \quad \text { and where } \Lambda=(L+1-s-p s+p r),
\end{aligned}
$$

whilst for $r \leq L<s$, we have (6a), and for $L<r, N_{r}(L)=0=N_{s}(L)$. Since $r<s$, the sums in (8) can be rewritten as

$$
2 p \sum_{n=0}^{L-r} N_{r}(n)+2(1-p) \sum_{n=0}^{L-s} N_{r}(n)=2 \sum_{n=0}^{L-s} N_{r}(n)+2 p \sum_{n=L-s+1}^{L-r} N_{r}(n) .
$$

Since, in practice, $L \gg r, s$, this means that the two sums over large numbers of terms are replaced by one sum over a large number of elements $(0 \leq n \leq L-s)$, and a second sum, which includes a much smaller number of terms $(L-s+1 \leq n \leq L-r)$.

We transform (8) by making the substitution $L \mapsto L+1$ and take the difference of the transformed equation and the original, together with the identity (10), to obtain

$$
\begin{aligned}
& 2 N_{r}(L-s+1)-N_{r}(L+1)+p \\
& =[L+1-s-p r+p s]\left[N_{r}(L+1)-N_{r}(L)\right] \\
& \quad+2 p\left[N_{r}(L-s+1)-N_{r}(L-r+1)\right],
\end{aligned}
$$

A similar method for $N_{s}(L)$ is used on (9), yielding

$$
\begin{aligned}
& 2 N_{s}(L-s+1)-N_{s}(L+1)+(1-p) \\
&= {[L+1-s-p r+p s]\left[N_{s}(L+1)-N_{s}(L)\right] } \\
&+2 p\left[N_{s}(L-s+1)-N_{s}(L-r+1)\right] .
\end{aligned}
$$

The equations (11)-(12), are formally uncoupled, however, solutions to both are required for a full understanding of this two-component RSA system. Whilst (11)-(12) holds for $L \geq s$, for $r \leq L<s$, we have just (6a) together with $N_{s}(L)=0$, and for $L<r$, $N_{r}(L)=0=N_{s}(L)$.

Given a solution for $N_{r}(L), N_{s}(L)$, we calculate the coverages as

$$
\theta_{r}=\frac{r N_{r}(L)}{L}, \quad \theta_{s}=\frac{s N_{s}(L)}{L}, \quad \text { and } \quad \theta=\theta_{r}+\theta_{s} .
$$


As $L$ increases from zero, clearly the first polymer allowed to bind is an $r$-polymer, which can only bind once $L \geq r$. Once $L$ is of greater length than $r$, there are two cases which need to be considered. The first is (a) the possible binding of an $s$-polymer, which first occurs when $L=s$; the second, (b), occurs when $L=2 r$, at which point it is possible for two $r$-polymers to bind, provided the first binds at one end of the substrate. In the special case $s=2 r$, both possibilities arise simultaneously at $L=s=2 r$. If $s<2 r$ then, as $L$ increases, case (a) occurs before (b), that is, $s$-binding occurs first; whereas if $s>2 r$, then case (b) occurs before (a), and multiple $r$-polymers can bind before a single $s$-polymer. Both scenarios will be considered in the following sections.

\section{Numerical simulations}

\subsection{Monte Carlo Simulations}

Monte Carlo simulations are extremely useful for modelling situations such as RSA. At each step in the simulation we choose either a long or short polymer, according the probability $p$, and attempt to land it on the substrate. If it finds a location of sufficient size, it binds to the substrate, making either $r$ or $s$ consecutive sites unavailable for binding. Once there are no stretches of free sites of length $r$ or greater, then the simulation stops, no more binding events are possible, and the substrate is jammed. A count of the number of binding events is used as an effective time. The simulation is carried out many times, and an average coverage is calculated, with larger numbers of simulations leading to increased accuracy.

All five graphs in figure 3 show the coverage increasing as successive binding events are attempted. The results show similarly shaped curves, converging to the same limit, though when the substrate length, $L$, increases, the system takes longer to reach the final jammed state. In the case illustrated, $p=0.2$, meaning that the shorter polymer has a lower probability of binding, and we note that the coverage by the shorter polymer takes a longer time to reach steady-state than the longer polymer. This is due to the short polymer filling in the gaps left by the longer polymer in the final stages of the transition to the jammed state.

We have performed simulations for a range of values for $p, r$, and $s$, recording the final coverage due to each polymer type. Graphs of coverage against $p$ agree with the results plotted in figure 5, which is discussed in section 3.2. Figure 6 shows the coverages $\theta_{*}$ plotted against substrate length, $L$, for the Monte Carlo simulations, and two theoretical techniques: a full solution of the recurrence relation, and an asymptotic approximation outlined later.

\subsection{Numerical solution of deterministic recurrence relation}

We observe approximately linear relationships between the numbers of each type of polymer bound and the substrate length, as in the one polymer case shown in the left panel of figure 2. In figure 4 we plot the coverage of each type of polymer, $\theta_{r}, \theta_{s}, \theta$, 


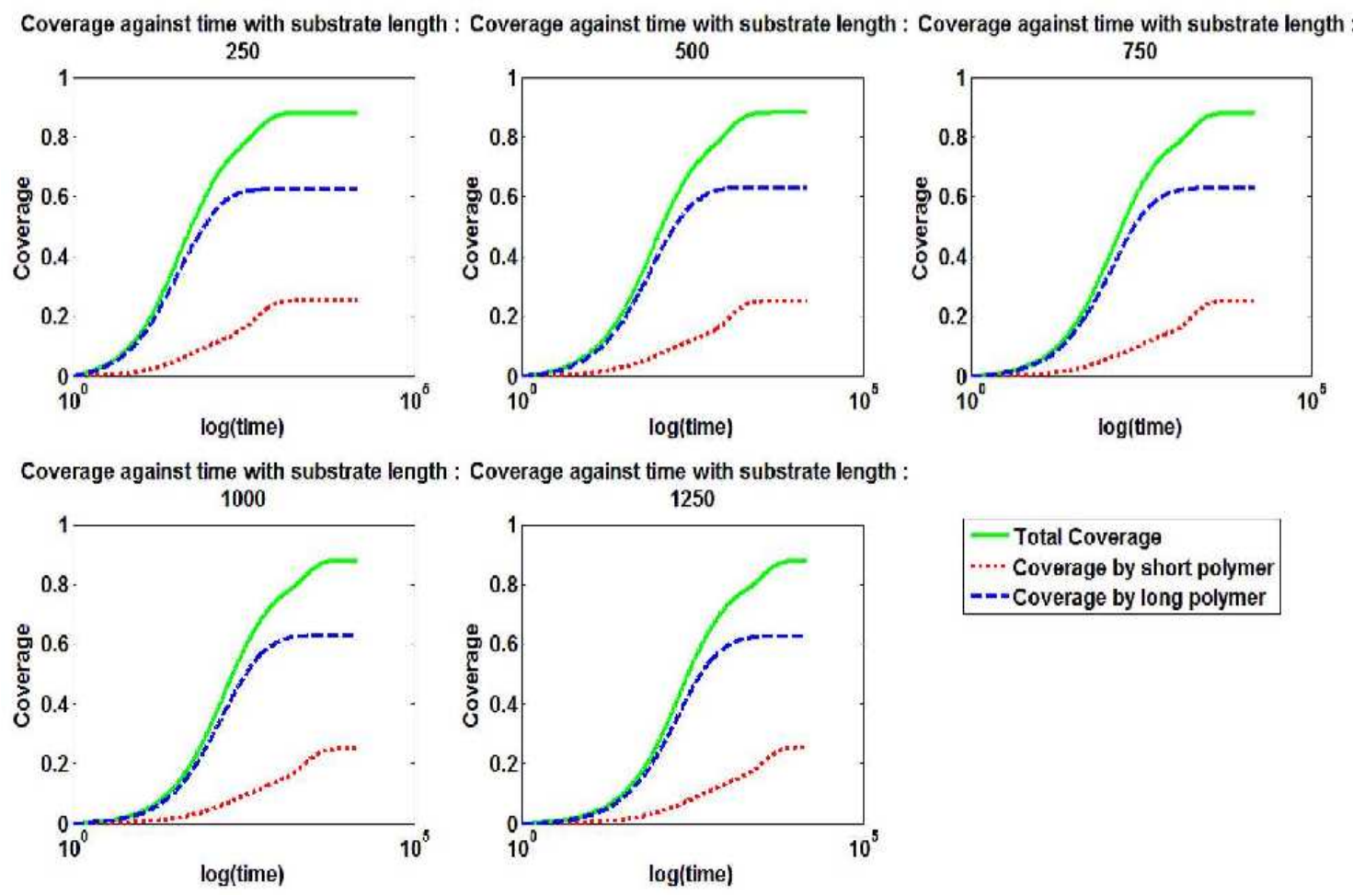

Figure 3. Graphs obtained using Monte Carlo simulations for different lengths of the substrate, showing how the coverage by the polymers changes as RSA proceeds. Parameter values: $r=3, s=5, p=0.2$, averages are taken over 1000 iterations.

against substrate length for a range of polymer length ratios and relative binding rates, corresponding to the right panel of figure 2. In the upper panels of figure $4, r=3$ and $s=5$, so that $r<s \leq 2 r$; whereas in the lower panels $r=3$ and $s=30$, so that $s>2 r$. On the left we have $p=0.2$ so the longer polymer binds at a higher rate, and on the right, $p=0.8$ so that the shorter polymer binds more rapidly.

In all cases the coverage due to the longer polymer, $\theta_{s}$ increase with substrate length, $L$, as in the one-polymer case. However, in most cases, the coverage due to the shorter polymer, $\theta_{r}$, decreases with increasing substrate length the dependence is not clear in the case $s=5, p=0.8$ (the top right panel). The total coverage, $\theta=\theta_{r}+\theta_{s}$ increases with substrate length in all cases. In the case of $r=3, s=5$ and $p=0.2$, the total coverage is $\theta=0.873$, when $r=3, s=5$ and $p=0.8$, we have $\theta=0.831$, thus the total coverage is higher when the probability of choosing the shorter polymer is lower.

Higher total coverages are also found when the ratio of long to short polymer lengths is increased, when $r=3, s=30$ and $p=0.2$, the total coverage reaches $\theta=0.918$. These higher coverages are achieved when $p$, the probability of the smaller polymer binding, is small, which leads to an initial period of more rapid deposition of longer polymers. On a slower timescale, there follows a slower stage in which the smaller polymers fill in the gaps between the larger polymers.

In figure 5 we plot the total coverage and the coverage due to each polymer species 

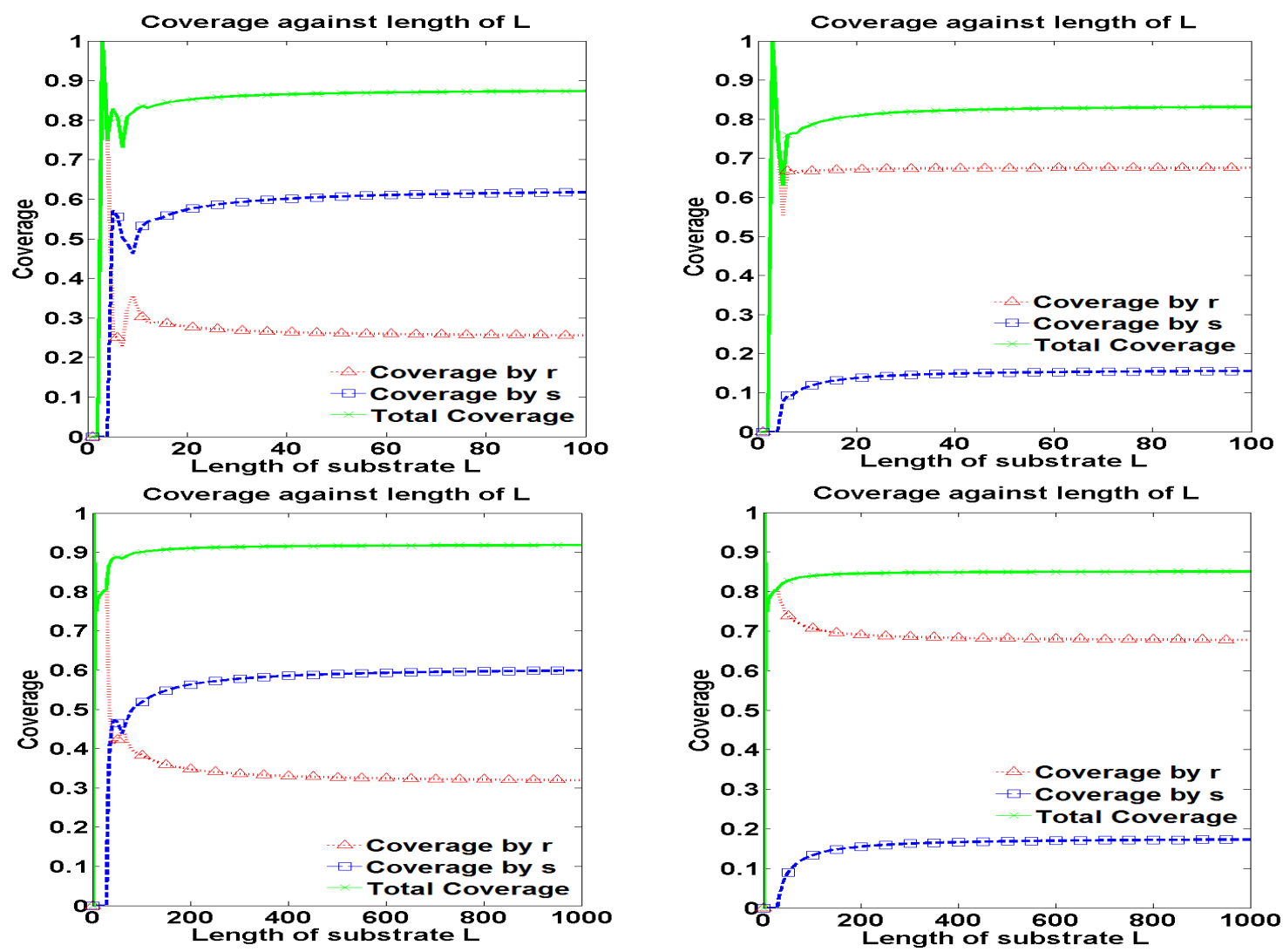

Figure 4. Expected coverages $\theta_{r}, \theta_{s}, \theta(13)$ calculated from the recurrence relations (11)-(12) plotted as functions of substrate length, $L$. Upper panels: the case of similar lengths of binding species, $r<s<2 r$; lower panels: the case of vastly differing lengths, $s>2 r$; left panels: the case of long species binding more rapidly than short; right panels: the case of short species binding more rapidly than long. Parameter values: top left: $r=3, s=5, p=0.2$; top right: $r=3, s=5, p=0.8$; lower left: $r=3, s=30, p=0.2$; lower right: $r=3, s=30, p=0.8$.

against probability of choosing the shorter polymer, $p$. On the left we show the results for the case of similar lengths $1<s / r<2$, and on the right, the case of vastly differing lengths, that is, $s / r>2$. The two graphs are qualitatively similar, and show an approximately linear dependence on $p$. There is a discontinuity at $p=0$ : when $p=0$, we have a one-polymer system, thus the maximum coverage will be about 75 $80 \%$. However, for $0<p \ll 1$, there are two polymers, and the longer one binds on a quicker timescale, and the shorter one on the slower timescale, filling in the gaps left by the longer polymer, thus leading to significantly higher coverages.

\section{Asymptotic Methods}

The final method we use to calculate the solution of the recurrence relations (11)-(12) is asymptotic approximations. We consider two cases for the relative magnitudes of $r$ and $s$ : (i) significant differences in the magnitudes of polymer lengths, that is, $r \ll s$ 


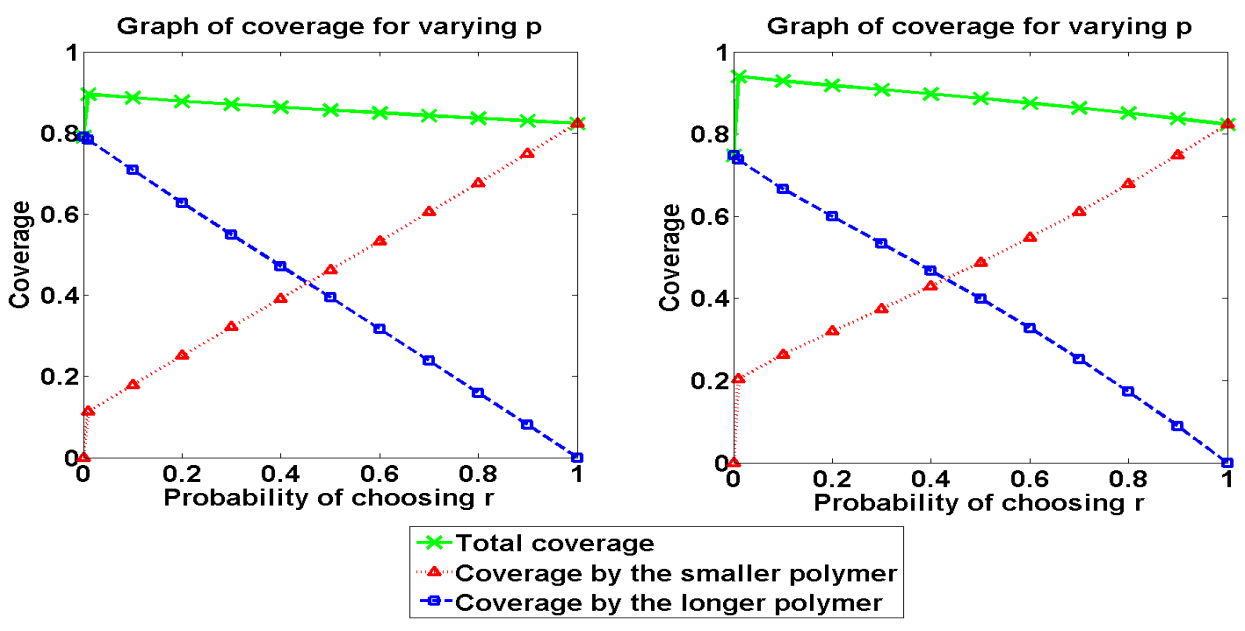

Figure 5. Graphs showing how the coverages of each polymer type changes with $p$, the probability of choosing $r$. Left, similar polymer lengths, $r=3, s=5$, and $L=1000$; right, vastly dissimilar polymer lengths, $r=3, s=30$ and $L=1000$.

with $1 / r \sim r / s \sim s / L \ll 1$; and (ii) similar lengths, that is, $r \sim s$. Case (ii) follows the theory outlined in [18] for the single component RSA; however, the addition of a second binding species significantly complicates the problem. In Case (i), we also follow the asymptotic approach of [18], but new scalings are required to account for the significantly different lengths of the binding species.

\subsection{Polymers of dissimilar length}

Clearly if $r \ll s$ then the scaling $r \sim s$ fails, so we require different asymptotic scaling from those used in [18]. Here, we consider the case where $r \sim s / r \sim L / s \gg 1$. So we replace the variables in the recurrence relation (11) with the variables

$$
r=\frac{1}{\epsilon}, \quad s=\frac{\widehat{s}}{\epsilon^{2}}, \quad L=\frac{y}{\epsilon^{3}}, \quad N_{r}(L)=\frac{1}{\epsilon^{2}} n_{r}(y), \quad N_{s}(L)=\frac{1}{\epsilon} n_{s}(y),
$$

where $\widehat{s}, y, n_{r}(y)$ and $n_{s}(y)$ are all $\mathcal{O}(1)$, with $\epsilon \ll 1$. This is due to our expectation that, for $0<p<1$, an $\mathcal{O}(1)$ amount of coverage will be due to each of the $r$ - and $s$-polymers, thus $r N_{r}=\mathcal{O}(L)$ and $s N_{s}=\mathcal{O}(L)$. We use primes to denote derivatives with respect to $y$. We substitute the ansatz (14) into the governing equation (11) and 
(12), to obtain

$$
\begin{aligned}
& \frac{2}{\epsilon^{2}} n_{r}\left(y-\epsilon \widehat{s}+\epsilon^{3}\right)-\frac{1}{\epsilon^{2}} n_{r}\left(y+\epsilon^{3}\right)+p \\
& =\quad\left[\frac{y}{\epsilon^{3}}+1-\frac{\widehat{s}}{\epsilon^{2}}-\frac{p}{\epsilon}+\frac{p \widehat{s}}{\epsilon^{2}}\right] \frac{1}{\epsilon^{2}}\left[n_{r}\left(y+\epsilon^{3}\right)-n_{r}(y)\right] \\
& \quad+\frac{2 p}{\epsilon^{2}}\left[n_{r}\left(y-\epsilon \widehat{s}+\epsilon^{3}\right)-n_{r}\left(y-\epsilon^{2}+\epsilon^{3}\right)\right], \\
& \frac{2}{\epsilon} n_{s}\left(y-\epsilon \widehat{s}+\epsilon^{3}\right)-\frac{1}{\epsilon} n_{s}\left(y+\epsilon^{3}\right)+(1-p) \\
& =\quad\left[\frac{y}{\epsilon^{3}}+1-\frac{\widehat{s}}{\epsilon^{2}}-\frac{p}{\epsilon}+\frac{p \widehat{s}}{\epsilon^{2}}\right] \frac{1}{\epsilon}\left[n_{s}\left(y+\epsilon^{3}\right)-n_{s}(y)\right] \\
& \quad+\frac{2 p}{\epsilon}\left[n_{s}\left(y-\epsilon \widehat{s}+\epsilon^{3}\right)-n_{s}\left(y-\epsilon^{2}+\epsilon^{3}\right)\right] .
\end{aligned}
$$

Next, we Taylor expand these equations using $\epsilon \ll 1$ which, after much cancellation, leads to

$$
y n_{r}^{\prime}(y)-n_{r}(y)=-\epsilon \widehat{s}(1-p) n_{r}^{\prime}(y)+\epsilon^{2}\left[\widehat{s}^{2}(1-p) n_{r}^{\prime \prime}(y)+p+p n_{r}^{\prime}(y)\right]+\mathcal{O}\left(\epsilon^{3}\right) .
$$

We note that (17) is inhomogeneous at $\mathcal{O}\left(\epsilon^{2}\right)$ but homogeneous at $\mathcal{O}(\epsilon)$.

Substituting $n_{r}(y)=A_{r}(\widehat{s}, p) y+\epsilon B_{r}(y, \widehat{s}, p)$ into (17) means that (17) is satisfied up to $\mathcal{O}(\epsilon)$ for any value of the arbitrary 'constant' $A_{r}$. Expanding and rearranging at next order in $\epsilon$ gives $y B_{r}^{\prime}(y)-B_{r}(y)=-(1-p) \widehat{s} A_{r}$, which can be solved using the integrating factor $1 / y^{2}$ to give $B_{r}=-(p-1) \widehat{s} A_{r}$, and hence $n_{r}(y)=A_{r} y-\epsilon(p-1) \widehat{s} A_{r}$. Substituting in the initial variables (14) into this solution, we obtain

$$
N_{r}(L)=\frac{A_{r} L}{r}\left[1+\frac{s(1-p)}{L}\right],
$$

where $A_{r}=A_{r}\left(s / r^{2}, p\right)$. Hence the coverage due to the shorter polymers is given by

$$
\theta_{r}(L ; s / r, p)=A_{r}\left(1+\frac{s(1-p)}{L}\right)
$$

which decreases with increasing $L$ for all values of $p$.

For the long polymers, Taylor-expanding (16) and simplifying gives

$$
y n_{s}^{\prime}(y)-n_{s}(y)=\epsilon(1-p)\left[1-\widehat{s} n_{s}^{\prime}(y)\right]+\epsilon^{2}\left[(1-p) \widehat{s}^{2}-p n_{s}^{\prime}(y)\right]+\mathcal{O}\left(\epsilon^{3}\right) .
$$

The different scalings of $r$ and $s, N_{r}$ and $N_{s}$ in (14) results in this differential equation for $n_{s}(y)$ is quite different to that for $n_{r}(y)$, namely (17). Substituting $n_{s}(y)=\widetilde{A}_{s}(\widehat{s}, p) y+\epsilon B_{s}(y ; \widehat{s}, p)$ into $(20)$ leads to $y B_{s}^{\prime}-B_{s}=(1-p)\left(1-\widehat{s} \widetilde{A}_{s}\right)$, which yields the solution $n_{s}(y)=\widetilde{A}_{s} y[1-\epsilon(1-p) \widehat{s} / y]-\epsilon(1-p)$. Substituting in the original variables using (14) and $\widetilde{A}_{s}=r^{2} A_{s} / s$, we obtain

$$
N_{s}(L)=\frac{A_{s} L}{s}\left[1+\frac{(1-p) s}{L}\right]-(1-p)
$$


Comparing (18) with (21), we note that there is an extra term at the end of (21) due to $(20)$ being inhomogeneous at $\mathcal{O}(\epsilon)$. The coverage due to the longer polymers is thus given by

$$
\theta_{s}(L ; s / r, p)=A_{s}-\frac{\left(1-A_{s}\right) s(1-p)}{L},
$$

which increases with substrate length, that is, as $L \rightarrow \infty$; this result is in contrast with that for $\theta_{r}$ (19). Combining (19) and (22), we obtain

$$
\theta(L ; s / r, p)=A-\frac{s(1-p)(2 A-1)}{L} .
$$

Hence the total coverage will always increase with substrate length, $L$, since the Rényi constant for the mixed system, $A=A_{r}+A_{s}$, must satisfy $A>1 / 2$.

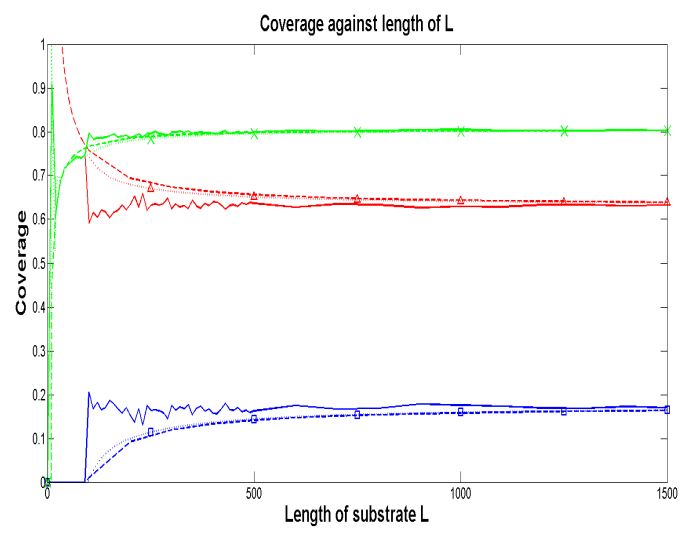

Asymptotics - Monte Carlo Numerics $\triangle$ Short polymers ${ }^{\circ}$ Long polymers $\times$ Total

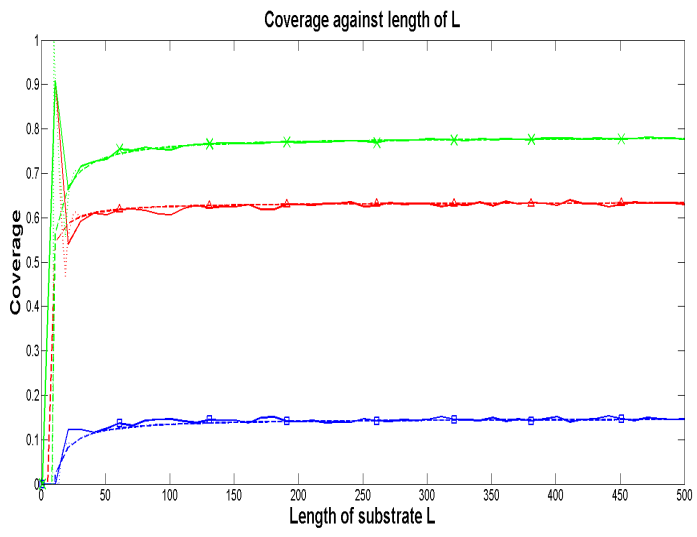

Asymptotics - Monte Carlo Numerics $\triangle$ Short polymers $\square$ Long polymers $\times$ Total

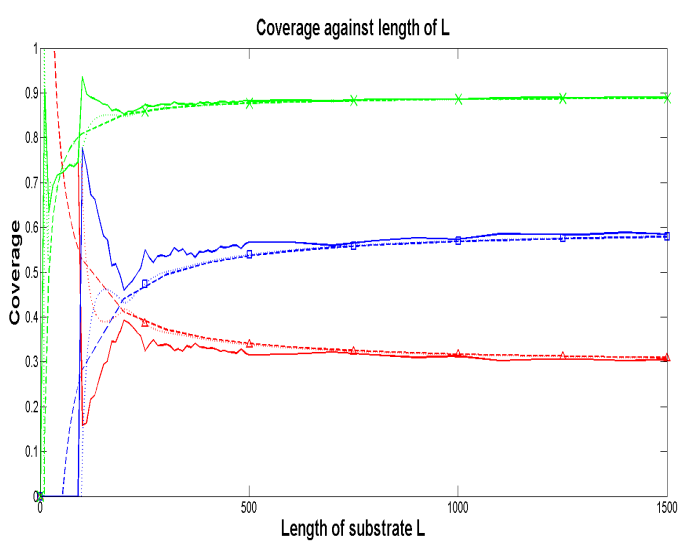

Asymptotics - Monte Carlo Numerics $\triangle$ Short polymers ${ }^{\square}$ Long polymers $\times$ Total

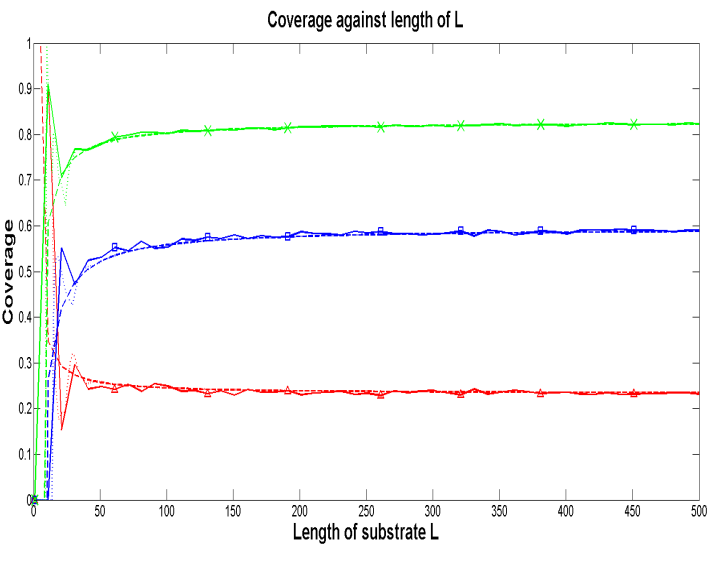

Asymptotics - Monte Carlo Numerics ${ }^{-S h o r t ~ p o l y m e r s ~}{ }^{\circ}$ Long polymers Total

Figure 6. Coverage plotted against substrate length for the case of massively different polymer lengths. Comparison of average of 100 Monte Carlo simulations with the asymptotic approximations (19), (22), and the numerical solution of the recurrence relations (11), (12). Upper left: $p=0.8, s=100$; upper right: $p=0.2, s=100$; lower left: $p=0.8, s=15$; lower right: $p=0.2, s=15$; in all cases $r=10$. 


\subsection{Assignment of constants, $A_{r}, A_{s}$}

In the asymptotic solutions (18) and (21), (and later in equations (30), (31)), the arbitrary constants, $\left.A_{r}(s / r, p), A_{s}(s / r, p)\right)$, are leading order approximations to the coverages $\theta_{r}, \theta_{s}$. From the graphs in figure 5, we note that the coverages are approximately linear in $p$, and by comparing the two graphs, for $s / r=5 / 3$ and $s / r=10$, we note that the dependence on $s / r$ is reasonably small.

We define $\phi_{r}$ to be Rényi constant for the discrete one-dimensional, one component system with polymer length $r$ in the limit $L \rightarrow \infty$. As a function of $p$, the coverage $\theta_{s}$ thus takes the value $\theta_{s}=\phi_{s}$ at $p=0$ and $\theta_{s}=0$ at $p=1$; hence $\theta_{s}=(1-p) \phi_{s}$. At $p=1, \theta_{r}=\phi_{r}$; however, in the limit $p \rightarrow 0^{+}$we have $\theta_{r}=\left(1-\phi_{s}\right) \phi_{r}$, since in this limit, the polymer will fill with $s$-polymers, leaving $\left(1-\phi_{s}\right)$ of the substrate free. Then a proportion $\phi_{r}$ of this will be filled with $r$-polymers. Hence we approximate $\theta_{r}$ by $\theta_{r}=p \phi_{r}+(1-p)\left(1-\phi_{s}\right) \phi_{r}$, and simplifying,

$$
A_{r}=\left(1-\phi_{s}+p \phi_{s}\right) \phi_{r}, \quad A_{s}=(1-p) \phi_{s}
$$

In figure 6 we compare all three of the methods used in this paper, namely, Monte Carlo simulations, a full numerical solution of the recurrence relations (11)-(12), and asymptotic solutions of these recurrence relations. The upper panels correspond to $r=10, s=100$, with $p=0.2$ and $p=0.8$. Clearly in both graphs, the results differ at small $L$ due to the asymptotic approximations being made on the assumption of $L \sim r^{3} \gg 1$. The asymptotic solutions and the numerical solution of the recurrence relations are difficult to distinguish on the two graphs. At larger values of $L$, all three solutions agree well. It is worth noting that in both graphs the coverage due to the longer species, $\theta_{s}$, and the total coverage both increase with $L$; whilst the coverage due to the shorter species, $\theta_{r}$ decreases with $L$. This is due to the fact that end effects cause a slight increase in the likelihood of shorter polymers binding.

\subsection{Polymers of similar length}

For this approximation, where $r$ and $s$ are similar in magnitude, and where $L \gg r$ we introduce a small parameter $\epsilon \ll 1$ defined by $\epsilon=1 / r$. Then we rescale the variables in the recurrence relation with new variables $\widehat{s}, y, n_{r}$, and $n_{s}$, all of which are $\mathcal{O}(1)$ and are defined by

$$
r=\frac{1}{\epsilon}, \quad s=\frac{\widehat{s}}{\epsilon}, \quad L=\frac{y}{\epsilon^{2}}, \quad N_{r}(L)=\frac{1}{\epsilon} n_{r}(y), \quad N_{s}(L)=\frac{1}{\epsilon} n_{s}(y) .
$$


Using these definitions, the governing equations (11) and (12) become

$$
\begin{aligned}
& \frac{2}{\epsilon} n_{r}\left(y-\epsilon \widehat{s}+\epsilon^{2}\right)-\frac{1}{\epsilon} n_{r}\left(y+\epsilon^{2}\right)+p \\
& =\quad\left[\frac{y}{\epsilon^{2}}+1-\frac{\widehat{s}}{\epsilon}-\frac{p}{\epsilon}+\frac{p \widehat{s}}{\epsilon}\right] \frac{1}{\epsilon}\left[n_{r}\left(y+\epsilon^{2}\right)-n_{r}(y)\right] \\
& \quad+\frac{2 p}{\epsilon}\left[n_{r}\left(y-\epsilon \widehat{s}+\epsilon^{2}\right)-n_{r}\left(y-\epsilon+\epsilon^{2}\right)\right], \\
& \frac{2}{\epsilon} n_{s}\left(y-\epsilon \widehat{s}+\epsilon^{2}\right)-\frac{1}{\epsilon} n_{s}\left(y+\epsilon^{2}\right)+(1-p) \\
& =\quad\left[\frac{y}{\epsilon^{2}}+1-\frac{\widehat{s}}{\epsilon}-\frac{p}{\epsilon}+\frac{p \widehat{s}}{\epsilon}\right] \frac{1}{\epsilon}\left[n_{s}\left(y+\epsilon^{2}\right)-n_{s}(y)\right] \\
& \quad+\frac{2 p}{\epsilon}\left[n_{s}\left(y-\epsilon \widehat{s}+\epsilon^{2}\right)-n_{s}\left(y-\epsilon \widehat{s}+\epsilon^{2}\right)\right] .
\end{aligned}
$$

In the following, we use prime to denote derivatives with respect to the rescaled substrate length variable, $y$. After expanding and much simplification, we obtain

$$
y n_{r}^{\prime}(y)-n_{r}^{\prime}(y)=\epsilon\left[(p \widehat{s}-p-\widehat{s}) n_{r}^{\prime}(y)+p\right]+\epsilon^{2}\left[\widehat{s}^{2}+p \widehat{s}^{2}-p-\frac{1}{2} y\right] n_{r}^{\prime \prime}(y) .
$$

Comparing (28) with (17), we note that in (28) we have gained an inhomogeneous term at $\mathcal{O}(\epsilon)$. Substituting the ansatz $n_{r}(y)=A_{r}(\widehat{s}, p) y+\epsilon B_{r}(y, \widehat{s}, p)$ into (28) leads to (28) being satisfied at leading order for any value of the arbitrary 'constant' $A_{r}$. At $\mathcal{O}(\epsilon)$, we have

$$
y B_{r}^{\prime}-B_{r}=(p \widehat{s}-p-\widehat{s}) A_{r}+p,
$$

which is solved by $B_{r}=-\left[(p \widehat{s}-p-\widehat{s}) A_{r}+p\right]$ and hence $n_{r}(y)=A_{r} y-\epsilon\left[(p \widehat{s}-p-\widehat{s}) A_{r}+p\right]$. Substituting back in the initial variables from (25), we find

$$
N_{r}(L)=\frac{A_{r} L}{r}\left[1+\frac{s(1-p)}{L}+\frac{p r}{L}\right]-p .
$$

The solution for $N_{s}$ can be obtained by symmetry arguments: mapping $p \rightarrow 1-p$, $r \rightarrow s, s \rightarrow r$ and $A_{r} \rightarrow A_{s}$, gives

$$
N_{s}(L)=\frac{A_{s} L}{s}\left[1+\frac{r p}{L}+\frac{s(1-p)}{L}\right]-(1-p) .
$$

Here $A_{r}=A_{r}(s / r, p)$ and $A_{s}=A_{s}(s / r, p)$ can be found from numerical solution of (11) for specific values of $p$ and $s / r$. Results of such calculations are shown in figure 5 for example values of $p, r, s$.

In the lower panels of figure 6 we show the coverage $\theta_{r}, \theta_{s}, \theta$ as functions of the substrate length for the case $r=10, s=15, p=0.2$ and $p=0.8$. We plot the asymptotic solutions for $\theta_{*}$ derived from (30), (31), the numerical solutions of (11)(12), and the average of 100 Monte Carlo simulations. At small lengths, where $L \sim r$, the asymptotic solutions are not accurate due to the asymptotic approximations being based on the assumption that $L \sim r^{2} \gg 1$. The case of smaller $p$ (lower right panel) yields a higher total coverage than larger $p$ (lower left) due to the substrate being 
predominantly covered by the larger polymers at early times, and the smaller polymers only binding in significant numbers when most of the gaps are of a size between $r$ and $s$. In the lower left panel, when $p=0.8$, small polymers land more frequently from the start, and the system rapidly reaches a state in which all gaps are smaller than $s$.

We observe that in the case of $p=0.8$ all curves $\theta_{r}, \theta_{s}, \theta$ rise with increasing $L$; just as in the one-component case analysed in Section 1.1. However, when $p=0.2$, increasing $L$ causes $\theta_{r}$ to decrease. We now use the formulae (24) to address this puzzling phenomenon.

\subsection{Reversal of asymptotic behaviour}

Calculation of the constants $A_{r}$ and $A_{s}(24)$ enables us to find the correction terms to $N_{r}(L), N_{s}(L)$, and hence, from (30)-(31), we find correction terms for the coverages $\theta_{r}$, $\theta_{s}$ as

$$
\begin{aligned}
& \theta_{r}=\left(1-\phi_{s}+p \phi_{s}\right) \phi_{r}+\frac{\phi_{r}}{L}\left[s(1-p)\left(1-\phi_{s}+p \phi_{s}\right)-p r\left((1-p) \phi_{s}+\frac{1-\phi_{r}}{\phi_{r}}\right)\right], \\
& \theta_{s}=(1-p) \phi_{s}-\frac{(1-p)}{L}\left[s\left(1-\phi_{s}\right)+(s-r) p \phi_{s}\right] \\
& \theta=1-\left(1-\phi_{r}\right)\left[1-(1-p) \phi_{s}\right]\left[1+\frac{p r+(1-p) s}{L}\right]
\end{aligned}
$$

The formulae for $\theta_{r}, \theta_{s}$ and $\theta$ all have the form $K_{0}-K_{1} / L$ : in the case of $\theta_{s}$ and $\theta$, the constant $K_{1}>0$, hence $\theta_{s}$ and $\theta$ always increase with $L$. However, in the formula for $\theta_{r}$, the constant $K_{1}$ can be positive or negative. For smaller $p, K_{1}<0$, and the curve $\theta_{r}$ decreases with increasing $L$, as observed in the lower right panel in figure 6 , and as seen in the case of vastly differing polymer lengths in Section 4.2. For larger $p, K_{1}$ may be positive, in which case $\theta_{r}$ increases with $L$, in a similar manner to $\theta_{s}$ and $\theta$, as in the lower left panel of figure 6 . This occurs if

$$
\frac{s}{r}<\frac{p\left[(1-p) \phi_{r} \phi_{s}+\left(1-\phi_{r}\right)\right]}{(1-p) \phi_{r}\left(1-\phi_{s}+p \phi_{s}\right)}
$$

The boundary between the two behaviours in $(p, s / r)$ parameter space is illustrated in figure 7 . Since we require $s>r$, only the area $s / r>1$ is relevant. In the majority of the region $\theta_{r}$ decreases with $L$ as in Section 4.2; however, in the smaller region, for $p$ closer to unity and smaller $s / r$ but still with $s / r>1$, we find $\theta_{r}$ increases with $L$.

\section{Conclusion}

We have analysed in detail the random sequential adsorption of two polymer lengths on a substrate, with varying rates, or probabilities, of binding. Our main interest is in how the coverage of the substrate at complete jamming changes as a function of the relative binding rates and length of substrate. A lower probability of choosing 


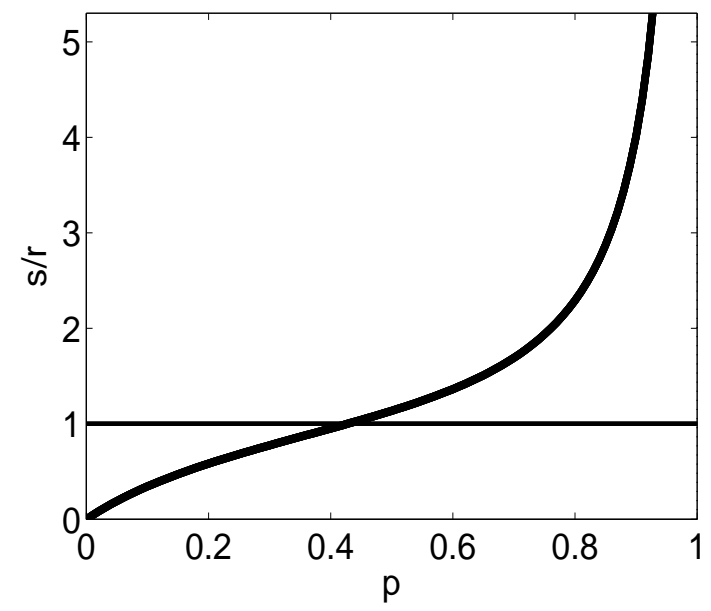

Figure 7. Illustration of the parameter space $(p, s / r)$. In the region below and to the right of the thicker line (35), and above the thinner line, both $\theta_{r}$ and $\theta_{s}$ increase with $L$; above the solid line, $\theta_{s}$ increases and $\theta_{r}$ decreases with substrate length, $L$.

the shorter polymer yields the greatest total coverage as then the large polymers land frequently, quickly filling the binding sites early in the process, and later, when there is no room for long polymers to bind, the shorter polymers are able to fill in the gaps. In this manner, coverages of over $90 \%$ can be achieved. We define the Rényi constant for shorter polymers by $\phi_{r}$ and that for longer polymers by $\phi_{s}$, then if there is a small probability of the shorter polymer binding, we rapidly find $\phi_{s}$ of the substrate covered, leaving $1-\phi_{s}$ vacant. If $r \ll s$, then over a longer timescale $\phi_{r}\left(1-\phi_{s}\right)$ of the substrate will be covered by the shorter polymer. This means the total coverage is $\phi_{s}+\phi_{r}-\phi_{r} \phi_{s}$. If we take the Rényi constant for the continuous system as a typical example, that is $\phi=\phi_{r}=\phi_{s}=0.7475979$, then we find a total coverage of $\phi(2-\phi)=0.936$.

We observe different asymptotic behaviour of coverages in the limit of large substrate lengths. In all cases, the total coverage and that of the longer polymer behave as in the one-component system, that is, as substrate length $L$ increases, so does the coverage, approaching a constant as the substrate length, $L \rightarrow \infty$, this limit being referred to as Rényi's constant. In the two component case, however, typically the coverage of the shorter polymer tends to its Rényi constant from above rather than below, thus the coverage due to the shorter polymer decreases with increasing $L$. If one were to divide the substrate of length $L$ into two of length $L / 2$, then we might expect a polymer to be cut, thus some rearrangement of polymers at the new ends would be required. If the broken polymer was short, then we have lower occupancy of the short polymers in the $L / 2$ substrates. However, if the broken polymer was long, then on at least one of the shorter polymers, there will be room for at least one short polymer, thus the coverage of short polymers may be more on the $L / 2$ substrates than the substrate of length $L$. This explanation clearly depends on the relative lengths of polymers and relative probabilities of them binding. However, this effect is not universal, as shown in figure 7 , there is a small region of parameter space, corresponding to more rapid 
binding of the shorter polymer and where the ratio of polymer lengths is not too large, in which all coverages increase with substrate length. Asymptotic analysis of the system has revealed conditions on the parameters under which each form of limiting behaviour holds.

In future work it would be interesting to investigate the binding of mixtures on two-dimensional substrates, to see if such more general systems exhibit similar or a wider variety of behaviours in their large size limits.

\section{Acknowledgements}

The work of LR was supported by a Wellcome Trust Biomedical Vacation Scholarship (2014) for which we are grateful. We thank Laura Davis and Nathan Bishop for performing preliminary numerical simulations. We are also grateful to the referee for helpful suggestions.

\section{References}

[1] Adamcyk Z and Weronski P 1998 Random sequential adsorption on partially covered surfaces. $J$. Chem. Phys. 1089851

[2] Baram A and Kutasov D 1992 Random sequential adsorption on a quasi-one-dimensional lattice: an exact solution. J. Phys. A: Math. Gen. 25 L493

[3] Bonnier B, Boyer D and Viot P 1994 Pair correlation function in random sequential adsorption processes. J. Phys. A; Math. Gen. 27 3671-3682

[4] Burridge DJ and Mao Y 2004 Recursive approach to random sequential adsorption Phys. Rev. E 69037102

[5] Cadilhe A, Araújo NAM and Privman V 2007. Random sequential adsorption: from continuum to lattice and pre-patterned substrates. J. Phys.: Condens. Matter 19065124

[6] Cornette V, Linares D, Ramirez-Pastor AJ and Nieto F 2007 Random sequential adsorption of polyatomic species. J. Phys. A: Math. Gen. 4011765

[7] Dias CS, Araújo, NAM, Cadilhe A 2012 Analytical and numerical study of particles with binary adsorption. Phys. Rev. E $\mathbf{8 5} 041120$

[8] Epstein IR 1979 Kinetics of large-ligand binding to one-dimensional lattices: theory of irreversible binding Biopolymers 18 765-804

[9] Evans JW 1993 Random and cooperative sequential adsorption. Rev. Mod. Phys. 65 1281-1329

[10] Feder J 1980 Random sequential adsorption. J. Theor. Biol. 87 237-254

[11] Flory P 1958 Intermolecular reaction between neighboring substituents of vinyl polymers. J. Am. Chem. Soc. 61 1518-1521

[12] Grosberg AY, Nguyen TT and Shklovskii BI 2002 Colloquium: the physics of charge inversion in chemical and biological systems. Rev. Mod. Phys. 74 329-345

[13] Hassan MK and Kurths J 2001 Competitive random sequential adsorption of point and fixed-sized particles: analytical results. J. Phys. A: Math. Gen. 347517

[14] Hassan MK, Schmidt J, Blasius B and Kurths J 2002 Jamming coverage in competitive random sequential adsorption of a binary mixture. Phys. Rev. E 65 045103(R)

[15] Hinrichsen EL, Feder J and Jøssang T 1986 Geometry of random sequential adsorption. J. Stat. Phys. 44 793-827

[16] Jian-Hong K, Xiao-Shuang C and Zhen-Quan L 2010 Analytical results for the cluster size distribution in controlled deposition processes. Chin. Phys. B 19026802. 
[17] MacLeod AS and Gladden, LF 1999 The influence of the random sequential adsorption of binary mixtures on the kinetics of hydrocarbon hydrogenation reactions. J. Chem. Phys. 1104000

[18] Maltsev E, Wattis JAD and Byrne HM 2006. DNA charge neutralisation by linear polymers: irreversible binding. Phys. Rev. E $\mathbf{7 4} 011904$.

[19] Maltsev E, Wattis JAD and Byrne HM 2006 DNA charge neutralisation by linear polymers, II: reversible binding. Phys. Rev. E, $\mathbf{7 4} 041918$

[20] McGhee JD and von Hippel PH 1974 Theoretical aspects of DNA-protein interactions: co-operative and non-co-operative binding of large ligands to a one-dimensional homogeneous lattice. J. Mol. Biol. 86 469-489

[21] Meyfroyt TMM, Borst SC, Boxman OJ, Denteneer, D 2014 A cooperative sequential adsorption model for wireless gossiping. arXiv:1407.6396

[22] Purves B, Reeve L, Wattis JAD, Mao Y 2015 Scaling behaviour near jamming in random sequential adsorption. Phys Rev E 91022118.

[23] Porschke P 1984 Dynamics of DNA condensation. Biochemistry 23 4821-4828

[24] Rényi A 1958 On a one-dimensional problem concerning random space-filling. Publ. Math. Inst. Hungar. Acad. Sci., 3 109-127

[25] Talbot J and Schaaf P 1989 Random sequential adsorption of mixtures. Phys. Rev. A 40, 422-427.

[26] Tarjus G, Schaaf P, and Talbot J. 1990 Generalized random sequential adsorption. J Chem Phys $938352-8360$

[27] Talbot J, Tarjus G, van Tassel PR and Viot P 2000 From car parking to protein adsorption: an overview of sequential adsorption processes. Coll \&3 Surf A: Physicochem \& Eng Aspects 165 287-324.

[28] Viot P, Tarjus G, Ricci SM, Talnbot J. 1992 Random sequential adsorption of anisotropic particles I jamming limit and asymptotic behaviour. J Chem Phys $\mathbf{9 7} 5212-5218$ 